\title{
Effects of a Slashpipe Training Intervention on Postural Con- trol Compared to Conventional Barbell Power Fitness
}

\author{
Nicolas Kurpiers $^{1 *}$, Teresa Rovelli ${ }^{1}$, Christin Bormann ${ }^{1}$ and Tim Vogler ${ }^{2}$ \\ ${ }^{1}$ Institute of Sport Science, University of Hildesheim, Germany \\ ${ }^{2}$ Orthopedic Surgeon and Sports Physician, Centre for Sports Medicine, Germany
}

*Corresponding author: Prof. Dr. Nicolas Kurpiers, Institute of Sport Science, University of Hildesheim, Endowed Professorship for Motion Science and Health Sports, Universitatsplatz 1, 31141 Hildesheim, Germany, Tel: 0049-(0)5121-88311918, Fax: 05121/883-11901, E-mail: kurpiers@uni-hildesheim.de

\begin{abstract}
Postural control is essential to maintaining balance and stability under health-related aspects. In the past several training concepts have been recommended for the enhancement of postural control. One of these concepts is the so-called 'Slashpipe' training, which uses a pipe partly filled with liquid in order to complete exercises similar to those found in barbell power fitness programs. However, evidence for the benefits of this intervention is rare. The aim of the study was to investigate the effects of a Slashpipe training intervention on balance ability. We recruited 31 individuals, eleven of which trained for 8 weeks using a Slashpipe (intervention group 1, IG-1), eight of which trained for 8 weeks using rigid weights (intervention group 2, IG-2), and eleven of which did not receive a training intervention (controls, CG). A Posturomed with a two-dimensional sensor was used before and after the 8-week period to measure single leg postural sway. The results showed a significant improvement for IG-1 and IG-2. The CG showed no change in postural sway. We therefore found evidence that the Slashpipe training did enhance stability. Under the current training regimen Slashpipe did not show improvements compared to conventional barbell power fitness exercises, however, this is the first study that revealed equal benefits for substantially different executions. Based on the findings in this study, Slashpipe might be an advantageous training tool to be complementary linked with other training types.
\end{abstract}

\section{Keywords}

Strength training, Stability, Posturomed

\section{Introduction}

The topic of core stability and postural control has increasingly gained awareness in diverse areas such as competitive and recreational sports, rehabilitation and prevention in the last two decades. The task of postural control involves controlling the body's position in space for (a) Stability (or balance) defined as the ability to maintain the body's projected Center of Mass (COM) within the limits of the Base of Support (BOS). It also involves (b) Orientation, defined as the ability to maintain an appropriate relationship between the body segments and between the body and the environment for a task [1]. Core stability is needed for postural control and describes the ability to hold tension in the abdominal and lumbopelvic region and a global and a local stability system distinguished. The global system comprises larger, more superficial muscles such as rectus abdominus and external obliques [2,3]. They are responsible e.g. for hip flexion and extension and trunk rotation. In contrast, the local stability system refers to deep, spine stabilizing muscles such as the transverse abdominus and multifidus that are responsible for the segmental stability of the lumbar spine and fine adjustments in postural control $[4,5]$. However, both systems merge in a synergistic relation as both systems are involved for any movement task that involves the trunk region [6]. Thus an appropriate approach for postural control and stability training needs to be found that incorporates the synergistic relation between the global and local stability systems and concurrently elicit satisfactory training effects [7]. 
Greater stability may benefit sports performance by providing a foundation for greater force production in the upper and lower extremities [8]. Meanwhile it is also common knowledge that a well-trained postural control can reduce the risk of lower extremity injuries [9-11]. Posture and balance as one of the most important motor skills for both everyday life and sport specific tasks involve not just the ability to recover from instability, but also the ability to anticipate and move in ways that will help you avoid instability [1].

Several training concepts were recommended with partly still insufficient scientific evidence, for example exercises on unstable equipment, such as Swiss Balls and BOSU balance trainer, improved static balance and postural control measures [12-15]. It is assumed that primarily functionally impaired patients would benefit from such improvements whilst benefits for athletes on performance measures partly still lack evidence.
Free weights exercises performed while standing on a stable surface are recommended for increases in core strength and power. Free weight exercises performed in that manner are specific to the core stability requirements of sports-related skills due to moderate levels of instability and high levels of force production $[8,16,17]$. Balance board and stability disc exercises, performed in conjunction with plyometrics exercises, are recommended to improve proprioceptive and reactive capabilities, which may reduce the risk of lower extremity injuries [18-22].

Glass and associates [23] investigated a specific training tool called 'Slosh Tube', which is a tube partly filled with water that produces instability due to the inertial changes in the device induced by water movement. The users hold it in their hands moving it according to specific exercises. Slight lateral movements lead to a redistribution of the liquid within the device which in turn cre-

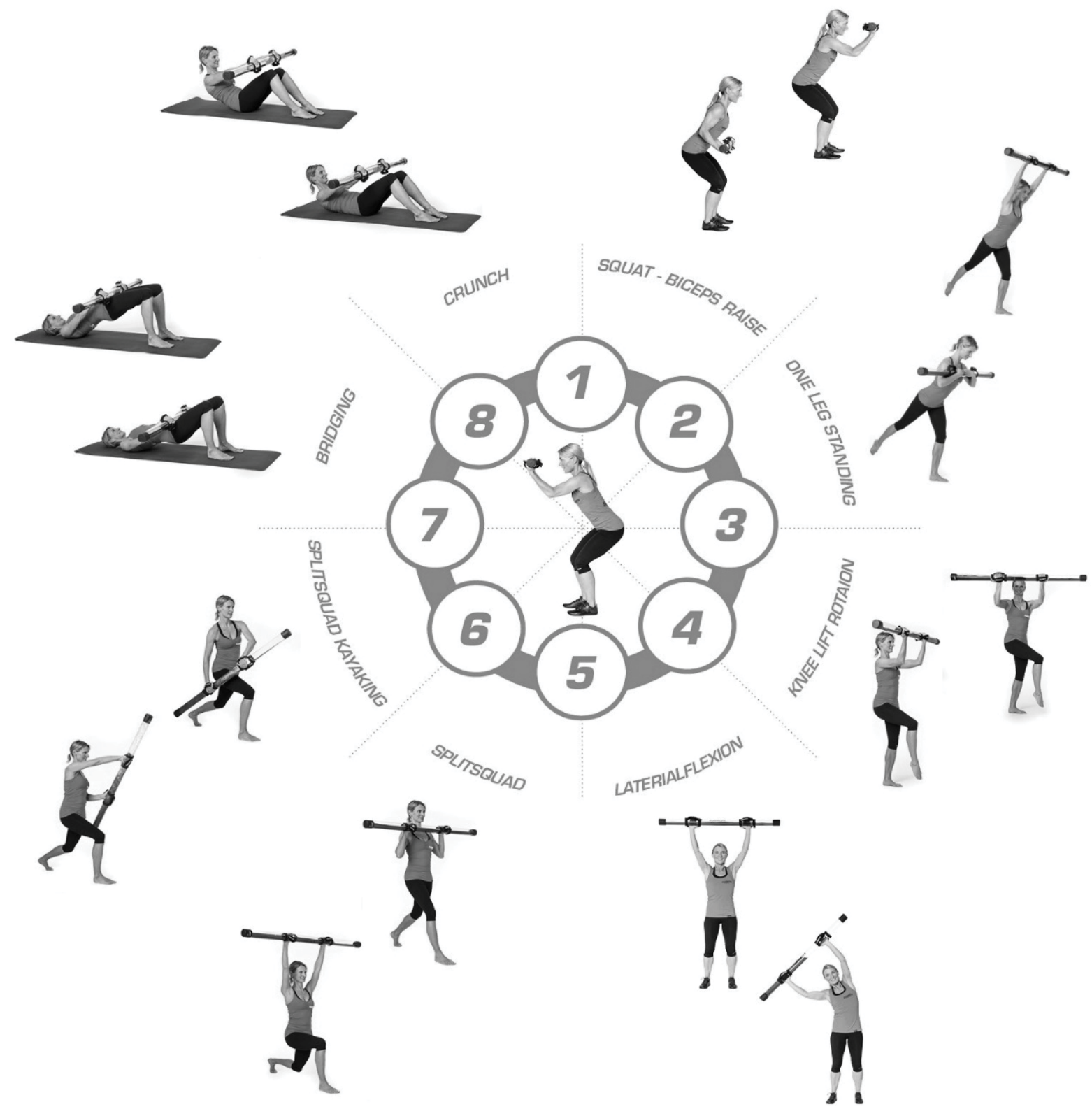

Figure 1: Slashpipe exercises. 
ates conditions where muscles need to compensate to hold the device steady while lifting. The authors found an increased variability of core muscle activation while performing a biceps curl using EMG measurements. The idea is that the instability caused by the tube leads to improvements in postural control.

The current study presents an investigation of a similar training device called 'Slashpipe', which has the same mechanical characteristics as the Slosh Tube. The inventors promise an increase in strength, flexibility, coordination, endurance and general capacity also for patients in clinical applications. A research group from Germany conducted an EMG based study on neck press, side tilt while standing, dead lifts and squats using a Slashpipe (Figure 1) and found an increased electromyographic activation (5\%) compared to rigid weights as well as increased activation peaks [24]. This was interpreted as a higher challenge to regulate muscle force and presumably means therefore an increase in coordinative demands. However, apart from the above reported effects evidence for the efficacy of this device compared to existing interventions remains unclear. The aim of the study was to investigate the balance abilities of a group undergoing a Slashpipe intervention and a group that trained with a stable barbell.

\section{Methods}

\section{Participants}

A total of 31 healthy adults (mean age 23 years, SD 2.46 years) participated in the study. The Slashpipe intervention group (IG-1) consisted of twelve participants ( 8 male, 4 female) with an average of 22.4 years ( \pm 3.34 $\mathrm{SD})$. Another intervention group (IG-2) consisted of eight participants ( 6 male, 2 female) with an average age of 22 years ( $\pm 1.51 \mathrm{SD}$ ) and a control group (CG) of another eleven participants ( 6 male, 5 female) with an average age of 24.4 years $( \pm 1.03$ SD).

All participants were informed about the aims of this study and everyone was free to leave the study at any time without justification. The ethics committee of the University of Hildesheim gave approval and all participants signed informed consent prior to the first test.

\section{Design and procedures}

The Slashpipe is similar to the previously described 'Slosh Tube', and is a pipe partly filled with water for several exercises in a standing, sitting, lying or kneeling position using straps for either the hands or feet (Figure 1). The so-called 'Power-Slashing interval' is the performance of instructed exercises in a group using the Slashpipe for intense intervals followed by less intense phases. A part of Power-Slashing is the Slashpipe Circuit Training (SCT), which is a training of 45 seconds of exercising followed by a 30 seconds break (or alternatively $30 / 15$ seconds on/off). Each exercise was performed in a pace of 125 beats per minute for three sets respective- ly. This intensity was given by the established Slashpipe SCT-Programme in order to regularly change between effort and relief. The training intervals had a specific order of exercises with different positions, movement velocities and joint angles to avoid local exhaustion [25].

The pre-defined SCT-Programme comprised the following exemplary exercises (Figure 1):

- Squat biceps raise

- One leg standing

- Knee lift rotation

- Lateral flexion

- Splitsquad

- Splitsquad kayaking

- Bridging

- Crunch

During these exercises, participants were advised to hold the pipe horizontally - as long as was appropriate for the respective exercise.

The participants of the IG-1 and the IG-2 both underwent an intervention of eight weeks, with training sessions occurring twice a week and each session lasting between 45 and 60 minutes. All training sessions were scheduled for Mondays and Thursdays between 10 and 12 o'clock. The difference between the IG-1 and IG-2 groups was the training gear, since the IG-1 performed all exercises using the Slashpipe whereas the IG-2 used a rigid barbell with the same weights, specifically $5.5 \mathrm{~kg}$ (Slashpipe ${ }^{\mathrm{TM}}$ Pro). The CG did not undergo any specific training program.

Coordination abilities were quantified by the measurement of balance which was tested twice, specifically on the day before the intervention started (pre-test) and after the eight weeks of the intervention (post-test). The tests for all three groups were performed in the same time scheme.

\section{Measures}

We assessed participant postural sway using a Posturomed (Haider Bioswing ${ }^{\circledR}$ ) and the Posturokybernetik-Test (Microswing 6 measurement system, Version 6.03.00) which is a standardized and evaluated procedure [26]. The participants performed a single leg stance on a swinging platform with a 2-dimensional sensor attached underneath.

The test involved the participant stepping onto the platform in a randomized order with the left and the right leg ten times respectively, with the participant holding a pre-defined position for ten seconds on each trial. The ability to regain balance was measured for both the left and right leg. The device calculated the mean values of the last eight trials, with the first two trials discarded because of the anticipated higher variability at the start 
Table 1: Results of the pre-intervention (pre) and post-intervention (post) balance test of the Slashpipe intervention group (IG-1), the Barbell intervention group (IG-2), and the passive control group (CG). Values given in percent, except for the P-values. Significant $\mathrm{P}$-values are indicated in bold.

\begin{tabular}{|l|l|l|l|l|l|l|}
\hline & \multicolumn{3}{l}{ Right Side } & \multicolumn{3}{l|}{ Left Side } \\
\hline & IG-1 $(\mathrm{n}=12)$ & IG-2 $(\mathrm{n}=8)$ & CG $(\mathrm{n}=11)$ & IG-1 $(\mathrm{n}=12)$ & IG-2 $(\mathrm{n}=8)$ & CG $(\mathrm{n}=11)$ \\
\hline Mean $\pm \mathbf{S D}_{\text {pre }}$ & $62.9 \pm 18.7$ & $64.6 \pm 6.4$ & $64.7 \pm 8.0$ & $65.7 \pm 7.51$ & $67.3 \pm 6.7$ & $64.4 \pm 6.9$ \\
\hline Mean $\pm \mathbf{S D}_{\text {post }}$ & $71.3 \pm 12.8$ & $70.8 \pm 4.94$ & $62.2 \pm 11.0$ & $71.8 \pm 7.5$ & $69.8 \pm 7.4$ & $62.9 \pm 9.2$ \\
\hline P-value (paired T-Test) & $\mathbf{0 . 0 2}$ & $\mathbf{0 . 0 0}$ & 0.16 & $\mathbf{0 . 0 1}$ & 0.11 & 0.6 \\
\hline Minimum value $_{\text {pre }}$ & 9 & 51 & 51 & 54 & 56 & 50 \\
\hline Minimum value $_{\text {post }}$ & 35 & 60 & 46 & 51 & 54 & 47 \\
\hline Maximum value $_{\text {pre }}$ & 78 & 72 & 80 & 79 & 78 & 79 \\
\hline Maximum value $_{\text {post }}$ & 80 & 75 & 80 & 80 & 81 & 77 \\
\hline
\end{tabular}

Table 2: P-values of paired T-Test of between-group comparison of the pre-intervention (pre) and post-intervention (post) balance test of the Slashpipe intervention group (IG-1), the Barbell intervention group (IG-2), and the passive control group (CG). Significant $\mathrm{P}$-values are indicated in bold.

\begin{tabular}{|l|l|l|l|l|l|l|}
\hline & IG_1 - IG_2 & IG_1 - CG & \multicolumn{3}{l|}{ IG_2 - CG } \\
\hline & right & left & right & left & right & left \\
\hline Pre-Test & 0.82 & 0.65 & 0.78 & 0.68 & 0.98 & 0.40 \\
\hline Post-Test & 0.92 & 0.56 & 0.10 & $\mathbf{0 . 0 2}$ & $\mathbf{0 . 0 7}$ & 0.12 \\
\hline
\end{tabular}

of the testing series. The output measures describe the relative stability on the swinging platform and are indicated in \%. Relative stability was assumed $100 \%$ when the person stands completely still, hence the lowest reachable stability measured by the Posturomed was $0 \%$ and the highest $100 \%$ [27].

\section{Statistical analysis}

Comparisons of the change in balance over the time period between the three groups (IG-1, IG-2 and CG) were calculated by one-way ANOVA while the comparison of the results of pre-test and post-test were analyzed with a paired $T$-test. $P$-values lower than 0.05 were considered significant. Statistical power was calculated using G-Power 3.1.9.2 with an effect size of 0.5 and a respective total sample size of 27 participants. All statistical analyses were calculated using IBM SPSS Statistics 23.

\section{Results}

\section{Posturokybernetic-test}

The IG- 1 achieved a stability increase of $8.4 \%$ on the right leg from the pre-test to the post-test with a statistically significant change for the pre-post-comparison $(p=0.02$, Table 1$)$. The mean values of the left legs changed significantly about $6.1 \%$ from the pre- to the post-test $(p=0.01)$.

The IG-2 had a right leg stability increase of $6.2 \%$ from the pre- to the post-test. The pre-post-comparison showed a significant change $(p=0.00)$ (Table 1). The left leg did not show any significant change (Table 1 ).

The balance of the CG on the right leg decreased slightly about $2.5 \%$ from the pre- to the post-test and thereby showed no significant change (Table 1). The left leg did not show any significance.

The comparison between the groups for the pretest showed no differences for both sides. The post-test differed significantly between IG-1 and CG for the left side and between IG-2 and CG for the right side whereas no differences are depicted between the intervention groups (Table 2).

\section{Discussion}

For the current investigation three different study groups were compared regarding the effects of an eight weeks training intervention on postural control. The training concept Slashpipe (IG-1) was compared to a conventional barbell power fitness program (IG-2) with the same weights and a group without specific training (CG). After eight weeks the IG-1 reached a significant increase in all balance values (left, right) whereas the IG-2 showed significant improvements for only the right leg. As assumed, the CG group did not show any substantial change after eight weeks. Both intervention groups (IG-1 and IG-2) differed significantly from the controls on one side (IG-1 left, IG-2 right) after the intervention whereas all groups showed no differences to each other in the pre-test.

Interpretation of the results in this study suggest positive effects of both the Slashpipe training and the barbell power fitness regarding postural control even though the stimuli for balance disturbances during the training sessions of the IG-2 were remarkably lower. It can be speculated that core muscles (which we did not measure) were enhanced for IG-1 and IG-2 which possibly led to an improvement in postural control after the intervention. However, as the IG-2 also reached significant results on one leg, it is not entirely clear whether the exercises itself (standing, challenging for coordination) or the pipe with its unsteady weight distribution led to the improvements. As the Slashpipe group performed only slightly better, it is possible that both interventions serve the mechanisms to enhance balance (e.g. core muscle activation, sensory motor activity), howev- 
er, without a clear difference. It also remains unclear whether this difference could increase with a longer intervention time and/or a greater sample size.

Freiwald [24] previously investigated Slashpipe exercises and found an increased electromyographic activation (5\%) compared to rigid weights as well as increased activation peaks. Glass, et al. [23] used EMG measurements in biceps curls with the 'Slosh Tube' and documented increased variability of core muscle activation meaning that core muscles under these circumstances were forced to adapt more in a compensatory way. The authors concluded that core muscles were more activated due to the internal random load redistribution and the turbulence within the device because of the flowing liquid. They also concluded that training with the water-filled tube was a sufficient stimulus to induce rapid and unstable adjustments of the muscles and attributed this effect to a potential prevention of injuries [23]. More indirectly, other researchers investigated spinal reflex times and cortical response times within neuromuscular training. It was found that a stimulus that is varied can improve the neuromuscular responsiveness of postural muscles and can lead to better postural control [28]. Earlier activation timings were also reached by performing a postural training using a vibrating platform [29].

Research also suggests that muscle adaptations during instability training are transferrable to improvements in strength in traditional stable resistance training, indicating that instability training can indeed contribute to increased strength $[30,31]$. Hence muscle adaptation during instability training due to improved postural control might lead to an economization of movements and thus the contracted muscle force can possibly better be focused on the sport-specific task rather than using a lot of it to compensate postural sway (in the above example strength in stable resistance training; in different contexts for example a shot on goal or preventatively bracing the leg to avoid injuries). This, however, needs to be proven by further studies with e.g. higher intensities, different combinations and sport-specific aspects within the measurements.

Postural adjustments are also activated before voluntary movements to minimize potential disturbances to balance that the movement may cause. This is called anticipatory postural control and may be the case in Slashpipe training, as moving the pipe causes (mainly lateral) disturbances that need to be countered. Sensory input (vision, vestibular system, proprioception) processed in the celebral cortex allows for improvements in stability to occur through refinements in neural programming [32].

It has specifically been reviewed that the developments of sensory systems and motor systems are reciprocally linked [33]. At the cortical level, motor maps are formed. Such motor maps can be attributed to areas of synaptic activity in the motor cortex in relation to specific tasks and get reorganized during the acquisition of a new motor task. It has been indicated that sufficient repetition of movement sequences is required to form and establish a motor map within the motor cortex [34]. For the Slashpipe performance it is questionable whether due to the repetitions a motor map gets formed and the motion pattern gets saved or if this is not possible as the motion pattern varies too much due to the steady redistribution of the liquid. Motor strategies and spine stabilizing muscles get apparently trained anyway which promotes postural control.

\section{Practical Applications}

We conclude that the Slashpipe concept specifically serve as an efficient additional training tool, to enhance balance and thus postural control for everyday life and specific sport tasks. It might be assumable that lifting light instable loads such as a Slashpipe could also be a promising option for clinical applications. Patients (e.g. with neuronal dysfunction or after cancer therapy) could initially use the instability to learn to rapidly activate muscles to balance during movements and thus gain or regain security. Additionally, the uniqueness of this training tool might arouse interest and motivation, particularly in young patients. This however, needs to be validated in follow up studies. It has to be shown in future studies whether combinations and different training intensities can also improve sport specific tasks.

\section{Acknowledgements}

We like to thank Stefan Flohr and Sebastian Gehlert for editing this article, Slashpipe $\mathrm{GmbH}$ for providing their gear and all participants for their contribution.

\section{References}

1. Shumway-Cook A, Woollacott MH (2001) Motor control: Theory and practical applications. Williams \& Wilkins.

2. Behm DG, Anderson K, Curnew RS (2002) Muscle force and activation under stable and unstable conditions. J Strength Cond Res 16: 416-422.

3. Bergmark A (1989) Stability of the lumbar spine. A study in mechanical engineering. Acta Orthop Scand Suppl 230: $1-54$.

4. Hodges P, Richardson C, Jull G (1996) Evaluation of the relationship between laboratory and clinical tests of transversus abdominis function. Physiother Res Int 1: 30-40.

5. McGill SM (2001) Low back stability: from formal description to issues for performance and rehabilitation. Exerc Sport Sci Rev 29: 26-31.

6. Cholewicki J, Vanvliet JJ (2002) Relative contribution of trunk muscles to the stability of the lumbar spine during isometric exertions. Clin Biomech 17: 99-105.

7. Marshall PW, Murphy BA (2005) Core stability exercises on and off a Swiss ball. Arch Phys Med Rehabil 86: 242-249.

8. Willardson JM (2007) Core stability training: applications to sports conditioning programs. J Strength Cond Res 21: 979-985. 
9. Borg G (1990) Psychophysical scaling with applications in physical work and the perception of exertion. Scand J Work Environ Health 16 Suppl 1: 55-58.

10. Leetun DT, Ireland ML, Willson JD, Ballantyne BT, Davis IM (2004) Core stability measures as risk factors for lower extremity injury in athletes. Med Sci Sports Exerc 36: 926-934.

11. Zazulak BT, Hewett TE, Reeves NP, Goldberg B, Cholewicki J (2007) The effects of core proprioception on knee injury a prospective biomechanical-epidemiological study. Am J Sports Med 35: 368-373.

12. Cosio-Lima LM, Reynolds KL, Winter C, Paolone V, Jones MT (2003) Effects of physioball and conventional floor exercises on early phase adaptations in back and abdominal core stability and balance in women. J Strength Cond Res 17: 721-725.

13. Scibek JS (2001) The effect of core stabilization training on functional performance in swimming. University of North Carolina at Chapel Hill.

14. Yaggie JA, Campbell BM (2006) Effects of balance training on selected skills. J Strength Cond Res 20: 422-428.

15. Silva PB, Mrachacz-Kersting N, Oliveira AS, Kersting UG (2018) Effect of wobble board training on movement strategies to maintain equilibrium on unstable surfaces. Hum Mov Sci 58: 231-238.

16. Baechle T, Earle R, Baechle TR (2004) NSCA's essentials of personal training. Human Kinetics.

17. Yessis M (2003) Using free weights for stability training Sticking to tried-and-true free weights for developing core strength may be the best solution. Fitness Management 19: 26-29.

18. Myer GD, Ford KR, Hewett TE (2004) Methodological approaches and rationale for training to prevent anterior cruciate ligament injuries in female athletes. Scand J Med Sci Sports 14: 275-285.

19. Myer GD, Ford KR, McLean SG, Hewett TE (2006) The Effects of Plyometric Versus Dynamic Stabilization and Balance Training on Lower Extremity Biomechanics. Am J Sports Med 34: 445-455.

20. Myer GD, Ford KR, Palumbo JP, Hewett TE (2005) Neuromuscular training improves performance and lower-extremity biomechanics in female athletes. Journal of Strength Conditioning Research 19: 51-60.

21. Myklebust G, Engebretsen L, Braekken IH, Skjølberg A, OI- sen OE, et al. (2003) Prevention of Anterior Cruciate Ligament Injuries in Female Team Handball Players: A Prospective Intervention Study Over Three Seasons. Clin J Sport Med 13: 71-78.

22. Paterno MV, Myer GD, Ford KR, Hewett TE (2004) Neuromuscular training improves single-limb stability in young female athletes. J Orthop Sports Phys Ther 34: 305-316.

23. Glass SC, Blanchette TW, Karwan LA, Pearson SS, O'Neil AP, et al. (2016) Core Muscle Activation During Unstable Bicep Curl Using a Water-Filled Instability Training Tube. J Strength Cond Res 30: 3212-3219.

24. Freiwald J (2014) Evaluation of Slashpipe. In: Wuppertal P, Bergische Universität Wuppertal.

25. Jablonowski F, Eckerl M (2017) Slashpipe.

26. Boeer J, Mueller O, Krauss I, Haupt G, Horstmann T (2010) Reliability of a measurement technique to characterise standing properties and to quantify balance capabilities of healthy subjects on an unstable oscillatory platform (Posturomed). Sportverletz Sportschaden 24: 40-45.

27. Microswing (2016) Handbuch für die Messsystemanwendung an Posturomed und Torsiomed.

28. Wojtys EM, Huston LJ, Taylor PD, Bastian SD (1996) Neuromuscular adaptations in isokinetic, isotonic, and agility training programs. American J Sports Med 24: 187-192.

29. Kennedy A, Bugnariu N, Sveistrup H (2013) Adaptation of the feedforward postural response to repeated continuous postural perturbations. Neuroscience and Medicine 4: 4549.

30. Cowley PM, Swensen T, Sforzo GA (2007) Efficacy of instability resistance training. Int J Sports Med 28: 829-835.

31. Langford GA, McCurdy KW, Ernest JM, Doscher MW, Walters SD (2007) Specificity of machine, barbell, and water-filled log bench press resistance training on measures of strength. J Strength Cond Res 21: 1061-1066.

32. Ruiz R, Richardson MT (2005) Functional balance training using a domed device. Strength and Conditioning Journal 27: 50-55.

33. Ostry DJ, Gribble PL (2016) Sensory Plasticity in Human Motor Learning. Trends Neurosci 39: 114-123.

34. Monfils MH, Plautz EJ, Kleim JA (2005) In search of the motor engram: motor map plasticity as a mechanism for encoding motor experience. Neuroscientist 11: 471-483. 